Running head: Structural priming in L2 comprehension

\title{
Lexicalized structural priming in second language online sentence comprehension
}

\author{
Hang $\mathrm{Wei}^{\mathrm{a}}$ \\ Julie E. Boland ${ }^{\mathrm{b}}$ \\ Jonathan Brennan $^{\mathrm{b}}$ \\ Fang Yuan ${ }^{\mathrm{a}}$ \\ Min Wang ${ }^{\mathrm{a}}$ \\ Chi Zhang ${ }^{\mathrm{c}}$ \\ ${ }^{a}$ Xi'an Jiaotong University, China \\ ${ }^{\mathrm{b}}$ University of Michigan, USA \\ ${ }^{\mathrm{c}}$ Ghent University, Belgium
}

Address correspondence to:

Hang Wei

School of Foreign Studies

Xi'an Jiaotong University

Xi'an, Shaanxi, 710049, China

Email: weihang2010@126.com 


\begin{abstract}
Prior work has shown intriguing differences between L1 and L2 comprehension priming of relative clauses. We investigated English reduced relative clause priming in Chinese adult learners of English. Participants of different education levels read sentences in a self-paced, moving window paradigm. Critical sentences had a temporarily ambiguous reduced relative clause. Across lists, critical sentences were rotated, so that they occurred either as prime or as target, and had either the same or different verb as the critical sentence with which they were paired. Prime/target pairs were separated by several filler sentences, which never contained a relative clause. Mean reading times for the disambiguating region in the target sentences were faster than in the prime sentences, but only in the same-verb condition, not in the different-verb condition. This pattern of results is consistent with L1 comprehension priming research, suggesting that similar lexically specific mechanisms are involved in L1 and L2 comprehension priming of reduced relative clauses. These findings are in line with lexicalist accounts of sentence comprehension (e.g., MacDonald et al., 1994), according to which syntactic information is bound to specific words. In addition, these findings argue against theories that postulate fundamental differences in processing of L1 and L2 (e.g., Clahsen and Felser, 2006a, 2006b).
\end{abstract}

\title{
Keywords
}

Sentence comprehension, structural priming, syntactic representations, relative clauses, second language 


\section{Introduction}

Language researchers have shown a growing interest in bilingual syntactic processing, partially driven by the ever-increasing population of second language (L2) speakers, and partially because this line of research can help to address important issues related to human language processing. For instance, is syntactic information represented in a similar way in the L2 as it is for native speakers of that language? Are the same mechanisms that are used in L1 processing also available to L2 processing, or is L2 processing qualitatively different from L1 processing (for reviews, see Clahsen and Felser, 2006b; Kaan, 2014)?

Structural priming provides a method for addressing these issues. It refers to the tendency to repeat or more easily process a target sentence because of its structural similarity to a previously encountered prime sentence (Bock, 1986). This tendency to repeat sentence structure allows researchers to explore how structural information is mentally represented, for when priming occurs, it indicates that the prime and target sentences are represented in a structurally related manner (Branigan and Pickering, in press). Structural priming has been extensively investigated among native speakers, initially in language production, and increasingly in language comprehension (for reviews, see Dell and Ferreira, 2016; Pickering and Ferreira, 2008). These studies have provided evidence for the use of abstract syntactic representations in L1 speakers, in that structural priming occurs in the absence of lexical and semantic overlap between prime and target in both passive and dative constructions (Bock, 1989; Bock and Loebell, 1990).

On the other hand, some constructions, such as English reduced relative clauses, produce measurable priming only when the prime and target share the same verb (e.g., Tooley et al., 2009), and most constructions show a lexical boost when the same verb is used (e.g., Hartsuiker et al., 2008; see Mahowald et al., 2016, for a recent review). As discussed below, the degree to which priming is lexically-dependent for a given construction has been taken as evidence that the grammatical knowledge used to process that construction is also lexically dependent. Thus, structural priming provides evidence about the very nature of grammatical representation. Investigations into priming in L2 learners should be similarly informative about the way syntactic information is represented in the second language, and whether L2 learners have similar or different representations of structural knowledge as do native speakers. However, as described below, the findings in L2 structural priming have been less consistent compared with L1 priming. 
L1 Relative Clause Priming. In a series of L1 experiments using various online reading methods, Traxler, Tooley and colleagues found that after reading a reduced relative clause (RC) like (1a), participants processed a subsequent reduced RC like (1b) faster in self-paced reading and eye-tracking studies (Traxler and Tooley, 2008; Traxler et al., 2014) and/or with reduced P600 in event-related potential (ERP) studies (Ledoux et al., 2007; Tooley et al., 2014; Tooley et al., 2009). The reduced RC structure was chosen because it is temporarily ambiguous between a main clause analysis (e.g., "the speaker" in 1a is doing the selecting) and a reduced relative clause analysis ("the speaker" is being selected). These options correspond to two different syntactic forms of the verb "selected", under lexicalist accounts (e.g., MacDonald et al., 1994). If readers initially adopt the main clause analysis, they will need to change to a relative clause interpretation during the prepositional phrase "by the group”.

(1a) The speaker selected by the group would be perfect for the program.

(1b) The architect selected by the manager was educated at Yale.

Compared with priming in production for passive and dative constructions, priming for relative clauses in comprehension seems more dependent upon lexical repetition between prime and target sentences. Thus, for RCs like (1a, 1b), reliable priming effects were observed when prime and target sentences contained the same embedded verb ("selected") but no priming occurred when prime and target contained different verbs (Ledoux et al., 2007; Tooley et al., 2009, 2014; Traxler and Tooley, 2008; though see Tooley and Bock, 2014 for an example of lexically independent relative clause priming). This finding has been taken as evidence for lexicalist accounts of sentence comprehension (e.g., MacDonald et al., 1994), according to which syntactic information is bound to specific lexical items such as verbs. On this account, syntactic priming should be enhanced when prime and target sentences contained the same verb but may not occur at all if prime and target contained different verbs ${ }^{1}$.

L2 Structural Priming. Structural priming has also been observed in L2 sentence comprehension. For example, Weber and Indefrey (2009) found lexically-dependent priming for L2 English passives among German L1 readers. At the same time, other researchers have found lexically independent priming for ambiguous relative clauses in L2 speakers of German and Italian, using a sentence-picture matching task (Nitschke et al., 2014).

Recently, Hui and Liu (2012) investigated structural priming during comprehension of L2 
relative clauses among Chinese-speaking learners of English in two self-paced reading experiments. In Experiment 1, prime and target contained the same verb (like 2a and 3), whereas in Experiment 2, prime and target contained different verbs (like $2 \mathrm{~b}$ and 3 ).

(2a) The rules changed by the Dean upset quite a few students. (Prime, same verb)

(2b) The child adopted by the couple was happy to have a home. (Prime, different verb)

(3) The policy changed after the accident annoyed many citizens. (Target)

Third-year undergraduates majoring in English (who started learning English in middle school around the age of 12, and had on average learned English for nine years) participated in the two experiments. Structural priming was observed in both experiments, suggesting lexical independence. More importantly, the finding of lexically-independent priming among L2 English learners stands in contrast to the lexically dependent priming observed for English native speakers reading reduced RCs (Ledoux et al., 2007; Tooley et al., 2009, 2014; Traxler and Tooley, 2008; Traxler et al., 2014). This inconsistency raises the intriguing possibility that RC priming in comprehension is served by lexicallyspecific mechanisms in L1, but lexically-independent mechanisms in L2. In other words, English reduced RCs may be represented more abstractly in L2 than in L1.

However, we know of no theory of L2 processing that suggests L2 syntactic representations should be more abstract than L1 representations. In fact, this finding stands in contrast to prior research on priming during L2 production using a variety of constructions, which suggests that lowerproficiency participants rely more on lexically specific syntactic representations, with abstract syntactic representations occurring only for the most proficient L2 learners (Bernolet et al., 2013; Kim and McDonough, 2008; Schoonbaert et al., 2007; for a review, see Hartsuiker and Bernolet, 2015).

It must be noted, however, that the experimental materials used in Hui and Liu (2012) are not strictly comparable to those used in L1 priming research (e.g., Tooley et al., 2009, 2014; Traxler and Tooley, 2008; Traxler et al., 2014). In particular, both the thematic role of the disambiguating region and the animacy of the head noun were less well controlled in Hui and Liu's study compared with the L1 priming research ${ }^{2}$. It is not clear to what extent these factors might have affected their results.

Our Experiment. Because the L2 priming results have been inconsistent with respect to lexical dependence, we ran a new experiment similar to Hui and Liu's (2012). We chose to also investigate the priming of RCs among Chinese-speaking learners of English for several reasons. First, L1 priming of relative clauses has been well-studied and numerous experiments have found support for lexically 
dependent priming (e.g., Tooley et al., 2009, 2014; Traxler et al., 2014). Second, Chinese and English belong to different language families, and the word order of relative clauses differs across the two languages: English RCs are head-initial, with the relative clause following the head noun (e.g., the man who is standing on the balcony), whereas RCs in Chinese are head-final, with the relative clause preceding the head noun (e.g., zhanzai yangtai shang de nage ren, standing on the balcony derelativizer the man). This makes it interesting to explore whether the typological difference between Chinese and English relative clauses might interfere with the processing of L2 relative clauses, as suggested in prior results (e.g., Nitschke et al., 2010). Cases with such a typological difference would seem to be exactly the cases where one would expect qualitatively different L1 and L2 processing.

To facilitate direct comparison with L1 priming results, we adopted materials from prior research on priming during L1 comprehension (e.g., Tooley et al., 2009, with minor modifications to suit our Freshman group participants, see Method for details). In addition, verb overlap was manipulated within-participants in the present study (cf. Hui and Liu, 2012), giving us more power to test the role of verb overlap in L2 priming.

Finally, prior research on priming in L2 production revealed that L2 syntactic representation develops with participants' language learning experience and proficiency, with lower-proficiency participants relying more on lexically specific syntactic representations (see Hartsuiker and Bernolet, 2015, for a review). To explore whether syntactic representations involved in L2 comprehension are also modulated by proficiency, we recruited participants of different L2 education levels (and proficiency), to be detailed in the following section.

\section{Method}

\section{Participants}

Two groups of Chinese-speaking learners of English participated in the experiment for partial course credit. The Freshman group consisted of 36 freshmen majoring in English at Xi'an Jiaotong University. Their average age was 19 years, and they had received formal English instruction in China for 6.5 years (starting at middle school around the age of 12) at the time of experiment. The Upperclassman group consisted of 34 students from the same university, including 20 juniors majoring in English (average age: 21; formal English leaning: 8.5 years) and 14 graduates majoring in applied linguistics (average age: 25; formal English learning: 10.5 years).

Apart from their L2 learning experience, a proficiency test was conducted among the participants 
prior to the experiment. The proficiency test was adopted from a prototype test for CET-4 (College English Test, Band 4), which had been used in a five-year validation study of CET among Chinesespeaking learners of English (Yang and Weir, 1998). Two out of the total five parts of the original test were used for the present study: a 30-item vocabulary and a 20 -item cloze, with a total score of $50^{3}$. The scores of the Upperclassman group $(M=38.28, S D=4.24)$ were higher than those of the Freshman group $(M=35.81, S D=4.53)$, as confirmed by an independent-samples $t$ test (two tailed), $t=2.25, d f=$ $62^{4}, p=.028$.

\section{Materials}

Sixty-four English reduced relative clauses were selected from a larger set used in prior research on structural priming during L1 comprehension (Tooley et al., 2009; See Appendix 1). These sentences were selected based on the vocabulary of our participants. Even so, in some cases, a word was replaced by an easier one, based on a pilot study conducted among students of the same education level as the Freshman participants. Each of the 64 sentences was rotated across four experimental lists. Each sentence appeared as a prime on two lists, and as a target on the other two lists (once with a same-verb prime and once with a different-verb prime, as shown in examples 4 and 5). Thus, on each list, half of the 32 prime-target pairs contained verbs that were repeated across prime and target sentences, and the other half contained different verbs. Each participant read only one version of each item.

(4a) The speaker selected by the group would be perfect for the program.

(4b) The architect selected by the manager was educated at Yale.

(5a) The director observed by the cop was in a bad part of the town.

(5b) The architect selected by the manager was educated at Yale.

Note that we used only one type of prime sentence, namely RCs, although a crossed design that involved both RC and main clause primes (MC, e.g., The customer selected a yellow jacket for his son) would be more effective in delineating structural priming from verb repetition effects (we return to this issue in Discussion). We adopted the current design because prior research on L1 speakers showed that the presence of alternative prime structures (e.g., MC) may delay the onset of structural priming effects in comprehension ${ }^{5}$. Thus, we follow several recent studies on comprehension priming in using only RC as the prime structure (Hui and Liu, 2012; Tooley et al., 2009, 2014) in order to obtain early priming effects and to allow for a direct comparison with prior results.

In addition, there were 162 filler sentences of various structures, including there-be construction 
(e.g., There is a wooden bridge over the river), simple transitive/intransitive sentences (She had been sitting there for a whole day), and complex sentences (Although I admire her courage, I don't think she acted wisely). None of the fillers involved the RC construction, and there was no content word overlap between the fillers and prime-target pairs. Each prime-target pair was separated by 2 to 6 fillers, with no fillers between the prime and the target. To encourage comprehension, 32 filler sentences were followed by a yes-no question (e.g., Is there a bridge over the river?). The answer to half of the comprehension questions was "yes".

\section{Procedure}

The experiment was conducted using E-prime software. Participants were tested individually and were randomly assigned to an experimental list. They were told that the goal of the study was to measure their reading speed and accuracy in comprehending English sentences, and hence they should read as quickly and carefully as possible. Participants were not informed of the research design until debriefing at the end of the study. They read sentences in a word-by-word, self-paced moving window display. At the beginning of each trial, the sentence appeared on the screen with all words covered by a single underline. Participants pressed "Enter" to view each consecutive word. At each press of the "Enter" button, the currently viewed word reverted to an underline as the next word was uncovered. The experiment began with a practice session comprising six additional fillers.

\section{Analyses}

The estimates of priming effects were based upon measuring participants' reading times on sentences like The architect selected by the manager was educated at Yale when it appeared as a prime versus when it appeared as a target. Recall that prime sentences were immediately preceded by unrelated filler sentences (see Materials for details). On the basis of prior results (e.g., Tooley et al., 2009, 2014; Traxler et al., 2014), we assumed that prime sentences themselves would not be primed by earlier prime and target $\mathrm{RC}$ sentences, because no priming effects have been found when an $\mathrm{RC}$ sentence was immediately preceded by another RC sentence that used a different verb.

Data were analyzed with linear mixed-effects regression modeling in R (e.g., Baayen et al., 2008) using the lme4 package (Bates, Maechler, et al., 2015). Following common practice in analyzing selfpaced reading data, we removed raw RTs that were below $100 \mathrm{~ms}$ and we replaced with the cutoff values any RT that was three standard deviations away from a participant's grand mean. These two measures together affected $2.5 \%$ of the data. To more closely satisfy the assumption of normality, we 
$\log$ transformed raw reading times for the statistical modeling. However, in the figures below we present the results in terms of the raw reading times.

Although the sentences were displayed one word at a time, for the purpose of analysis, we followed the practice of segmenting sentences into scoring regions (e.g., Tooley et al., 2014). For multi-word scoring regions, reading times were summed across the words. Three scoring regions were analyzed. The Verb region consisted of the verb inside the relative clause (e.g., selected). The PP region consisted of the prepositional phrase (e.g., by the manager), and the post-PP region consisted of the two words immediately following the PP region (e.g., was educated). Regions containing a removed value (below $100 \mathrm{~ms}$ ) were excluded on a trial by trial basis. Based on prior research (Tooley et al., 2009, 2014), we expected to see lexical repetition priming effects at the verb and syntactic priming effects in the disambiguating region.

The critical predictors in the models were priming (categorical predictor: prime vs. target), verb repetition (categorical predictor: same vs. different), and education level (categorical predictor ${ }^{6}$ : freshman vs. upperclassman). In addition, we also included the length (in letters) of each region (numerical predictor, created by summing the length of each word within each region), comprehension question accuracy (numerical predictor, computed across all stimuli with questions), stimulus order, which represents the order at which each experimental stimulus appeared in the list (numerical predictor, divided by 100 , so that all predicators were on a similar scale). To reduce collinearity, all categorical predictors were centered using contrast coding (e.g., prime $=-0.5$, target $=0.5$; same verb $=$ -0.5 , different verb $=0.5)$, and all numerical predictors were mean-centered. For data sets of this size, effects are considered significant at an absolute $t$-value of 1.96 or greater (Fine and Jaeger, 2016).

The random effects structure was determined following the back-off procedure described in Bates, Kliegl, et al. (2015). We started the analysis with a maximal model including by-subjects and by-items random intercepts and random slopes for the critical predictors indicating priming, verb repetition and education level and interactions between these predictors. To address problems with convergence, we backed-off, first, to a model without random correlation parameters, and then sequentially removed terms with zero variance. After achieving convergence, we added back in correlation parameters for the remaining variance components. The procedure was first conducted on the Verb region and then checked against the $P P$ and post- $P P$ regions. The final model for the Verb and post- $P P$ regions included random intercepts for participants and items, a by-participants random slope for the priming- 
by-verb repetition interaction, and by-items random slopes for priming, verb repetition, and their interaction. The final model for the $P P$ region was identical except lacked a by-participants random slope for the priming-by-verb repetition interaction. See Appendix 2 for details of the analyses and model-fitting procedure.

\section{Results}

\section{Comprehension question accuracy}

Four participants in the freshman group were excluded from further analyses because they scored below the exclusion cutoff of $80 \%$ accuracy in answering the comprehension questions. The remaining 32 participants had an average accuracy of $90.2 \%(S D=5.31 \%)$. In the upperclassman group, two participants were excluded from further analyses because of accuracy below $80 \%$. The remaining 32 participants scored an average of $90.2 \%(S D=5.65 \%)$. There was no difference in accuracy rates between the two groups $(t<1)$.

\section{Mean reading times}

Figure 1 presents mean reading times for the verb region. As shown in Figure 1, participants in both groups read the verb faster in the target sentence than in the prime sentence, especially when the verb was repeated between the prime and target sentences. This is borne out by the model that was fit to the verb region, which shows a main effect of priming $(\beta=0.047, S E=0.016, t=2.917)$, and an interaction between priming and verb repetition $(\beta=-0.074, S E=0.034, t=-2.179)$. We conducted two post-hoc tests to follow up on the interaction between priming and verb repetition, and the results show that the priming effect is reliable when the verb was the same between prime and target $(\beta=0.084, S E$ $=0.024, t=3.545)$ but not when the verbs were different $(\beta=0.01, S E=0.023, t=0.431)$. During this region, the sentence could be continued with either a main clause interpretation or a relative clause interpretation. Thus, effects in this region are probably due to lexical repetition of the verb. The modeling results also reveal a main effect of $\log$ stimulus order $(\beta=-0.133, S E=0.022, t=-6.15)$, indicating that participants tended to read faster as the experiment progressed. This effect, however, only emerged as a main effect and did not interact with priming $(\beta=-0.011, S E=0.023, t=-.456)$. A robust main effect of region length was also found $(\beta=0.066, S E=0.005, t=13.55)$, indicating that participants tended to spend more time as the length of the verb increased. No other effects reached significance. Results of the model fit to each region are summarized in Table 1. 
--------[insert Figure 1 about here]--------

Table 1. Coefficients, standard error $(S E)$, and $t$ values for each predictor at each sentence region

\begin{tabular}{|c|c|c|c|c|c|c|c|c|c|}
\hline \multirow[b]{2}{*}{ Predictor } & \multicolumn{3}{|c|}{ Verb } & \multicolumn{3}{|c|}{ PP region } & \multicolumn{3}{|c|}{ Post-PP region } \\
\hline & $\beta$ & $S E$ & $t$ & $\beta$ & $S E$ & $t$ & $\beta$ & $S E$ & $t$ \\
\hline Education level & -.019 & .073 & -.261 & .008 & .052 & .148 & .013 & .05 & .252 \\
\hline Priming & .047 & .016 & 2.917 & .029 & .008 & 3.5 & .002 & .01 & .208 \\
\hline Verb repetition & -.001 & .02 & .026 & .006 & .009 & .695 & .003 & .013 & .232 \\
\hline Region length & .066 & .005 & 13.35 & .014 & .002 & 6.684 & .023 & .002 & 13.49 \\
\hline Stimulus order & -.134 & .022 & -6.15 & -.119 & .013 & -9.395 & -.114 & .014 & -8.334 \\
\hline Accuracy & -.44 & .663 & -.664 & -.375 & .478 & -.785 & -.314 & .466 & -.674 \\
\hline Priming*Education & -.01 & .023 & -.439 & .002 & .014 & .174 & -.003 & .016 & -.193 \\
\hline Priming $*$ Verb repetition & -.074 & .034 & -2.179 & -.045 & .016 & -2.772 & -.017 & .023 & -.76 \\
\hline Priming*Stimulus order & -.011 & .023 & -.456 & -.012 & .011 & -1.07 & .011 & .013 & .809 \\
\hline $\begin{array}{l}\text { Education*Verb } \\
\text { repetition }\end{array}$ & .008 & .023 & .342 & .010 & .014 & .734 & -.002 & .016 & -.149 \\
\hline $\begin{array}{l}\text { Priming*Education* } \\
\text { Verb repetition }\end{array}$ & .049 & .047 & 1.049 & .012 & .028 & .43 & .026 & .034 & .762 \\
\hline
\end{tabular}

Note: Verb refers to the ambiguous verb (e.g., selected), $P P$ region refers to the disambiguating prepositional phrase (e.g., by the manager), and Post-PP refers to the two words immediately following PP (e.g., would be). For data sets of this size, $t$ values with an absolute value of 1.96 are considered significant (Fine \& Jaeger, 2016). Significant effects are in bold.

Mean reading times for the critical disambiguating PP region are shown in Figure 2. Targets were again read faster than primes $(\beta=0.029, S E=0.008, t=3.5)$. As revealed in Figure 2, the priming effect seems to be driven mainly by the verb-repetition condition. This was confirmed by the modeling results (see Table 1), which reveal a significant interaction between priming and verb repetition $(\beta=-$ $0.045, S E=0.016, t=-2.772$ ). Post-hoc tests show that there is reliable priming when the verb was repeated between prime and target sentences $(\beta=0.051, S E=0.01, t=5.092)$ but not when the verbs were different $(\beta=0.006, S E=0.013, t=0.492)$. There was also a main effect of log stimulus order $(\beta$ $=-0.119, S E=0.013, t=-9.395)$, but this effect did not interact with priming $(\beta=-0.012, S E=0.011, t$ $=-1.07)$. Hence, there was no evidence of priming effects accumulating from one trial to the next. The main effect of region length was also significant $(\beta=0.014, S E=0.002, t=6.684)$. No other effects reached significance. In particular, there was no effect of education level $(\beta=0.008, S E=0.052, t=$ $0.148)$ nor a three-way interaction between education level, verb repetition, and priming $(\beta=0.012, S E$ $=0.028, t=0.43)$.

--------[insert Figure 2 about here]-------- 
results only show the main effects of region length $(\beta=0.023, S E=0.002, t=13.49)$ and stimulus $\operatorname{order}(\beta=-0.114, S E=0.014, t=-8.334)$. No other effects reached significance.

--------[insert Figure 3 about here]--------

\section{Discussion}

The present study explored the extent to which L2 sentence processing is similar to L1 sentence processing by investigating priming of English L2 reduced relative clauses during comprehension. Two groups of Chinese-speaking learners with different English education levels participated in a self-paced reading experiment, in which each $\mathrm{RC}$ target sentence was immediately preceded by an $\mathrm{RC}$ prime that contained either the same verb or a different verb. Robust priming effects occurred at the disambiguating region when prime and target contained the same verb but not when prime and target contained different verbs. This pattern of results is comparable to that observed in L1 comprehension priming research (Tooley et al., 2014; Tooley et al., 2009; Traxler and Tooley, 2008; Traxler et al., 2014; cf. Tooley \& Bock, 2014), suggesting that similar lexically-specific mechanisms are involved in L1 and L2 comprehension priming for sentences with reduced relative clauses, despite the typological differences between L1 (Chinese) and L2 (English) relative clauses.

Before discussing the implications of the current results, we must address an issue related to the priming effect observed in this study: Does the facilitation effect in the disambiguating by-phrase region reflect structural priming as intended, or is it simply spillover from lexical-level repetition priming due to the repeated verb? ${ }^{7}$ Given that priming was most evident when the verb was repeated between the prime and target sentences, it is possible that the priming effect in the disambiguating region was actually caused by the repeated verb. However, prior research using exactly the same type of sentences (and similar design) as the present study suggests that the facilitation effect in the disambiguating region should not be interpreted as lexical priming. For instance, prior eye-tracking and ERP studies on L1 comprehension priming of RCs showed that repeating the verb itself without at the same time repeating the syntactic structure did not lead to priming at the disambiguating region: the disambiguating by-phrase of an RC target sentence (e.g., The architect selected by the manager was educated at Yale) was not read faster following a main clause prime that contained the same verb (e.g., The group selected the speaker who gave a great talk), though there was repetition priming at the verb 
(Ledoux et al., 2007; Traxler \& Tooley, 2008). Moreover, prior ERP studies showed that the facilitation effect at the repeated verb was different in nature from that effect in the disambiguating region, with the former reflecting facilitation of semantic integration (reducing the amplitude of the N400) while the latter reflecting syntactic facilitation effect (reducing the amplitude of the P600) (Tooley et al., 2009, 2014).

While effects in the moving window paradigm are sometimes delayed relative to ERP effects, these findings suggest that the facilitation effect observed in the disambiguating region should not be attributed entirely to verb repetition. Of course, given the ubiquity of priming effects at various levels (Pickering \& Ferreira, 2008), it's difficult to completely rule out any contribution from lexical repetition. Future experiments using a crossed design that involves both $\mathrm{RC}$ and main clause as prime structures will be needed to delineate the effects of structural priming from the lexical spillover effects.

The present findings are difficult to reconcile with current L2 sentence processing models such as the Shallow Structure Hypothesis (SSH, Clahsen and Felser, 2006a, 2006b), according to which L2 processing of complex structure is fundamentally different from L1 sentence processing. The SSH draws upon the distinction between grammatical relations that involve local dependencies (typically involving adjacent words or constituents) and those that involve nonlocal dependencies (as found in English $w h$-questions). Specifically, SSH predicts that L2 speakers can achieve native-like processing in the domain of local dependencies "but that L1 and L2 processing differences persist in the domain of complex syntax, even in highly proficient L2 speakers" (Clahsen and Felser, 2006b, p.564).

For English reduced relative clauses such as The speaker selected by the group would be perfect for the program, the NP "the speaker" has been moved from the relative clause to the subject position of the main clause (as illustrated in (6)), crossing the boundary of a local clause. This forms a nonlocal dependency between "the speaker" and its lexical subcategorizer "selected".

(6) [The speaker [(who was) selected___ by the group] would be perfect for the program].

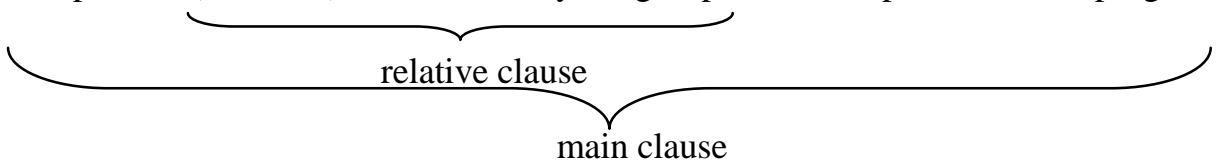

Our results indicate, however, that even for RCs that involve nonlocal dependencies, the same lexicalized mechanisms that are used in L1 comprehension priming are also involved in L2 comprehension priming, arguing against a fundamental difference between L1 and L2 sentence processing. 
Our results are consistent with prior results that revealed similar mechanisms underlying L1 and L2 online sentence comprehension. For instance, in a series of studies using both offline and online measures, Hopp $(2010,2016)$ showed that non-native and native grammars and processing systems are fundamentally the same, with L2 systems being constrained by factors such as L1 influence and/or word frequency. In addition, there is evidence that when their two languages are closely related, bilinguals are able to anticipate upcoming words in a similar manner as do monolinguals (e.g., Foucart et al., 2014; see Kaan, 2014, for a review).

The current finding of lexically-dependent priming is in conflict with Hui and Liu (2012), who found syntactic priming effects even in the absence of verb repetition between prime and target sentences. The reason for this inconsistency is not clear but might be due to the greater variability of Hui and Liu's experimental materials (see Note 2 for details), which in turn could have led their participants to rely more on abstract syntactic cues during comprehension, giving rise to the lexicallyindependent priming.

The present results did not find any effect of L2 learning experience on comprehension priming of the RC, with both freshman and upperclassman groups exhibiting lexically-dependent priming only. The prediction that L2 proficiency should modulate priming effects comes mainly from experiments on priming in production, where the effect of structural priming has been shown to undergo a developmental shift from lexically-dependent to relatively abstract priming with the increase of L2 proficiency (e.g., Bernolet et al., 2013; Kim and McDonough, 2008; Schoonbaert et al., 2007). Our two groups were too similar to adequately test this prediction. Thus, it is possible that more advanced L2 learners may demonstrate relatively abstract, lexically-independent priming in comprehension. However, the fact that English native speakers also show the lexically-dependent pattern (Tooley et al., 2009, 2014; Traxler et al., 2014; Traxler and Tooley, 2008; cf. Tooley and Bock, 2014) suggests that the pattern we observed for both education levels may in fact be the mature pattern for L2 learners.

The fact that we observed lexicalized priming only might be due to the reduced relative clause structure employed in the current study. As Tooley et al. (2014) suggest, the degree to which lexically independent priming could occur might be influenced by the relative strength of the different structure representations. English reduced RCs are temporarily ambiguous between relative clauses and other structural alternatives (e.g., main clause, passive) that are more frequent and less syntactically complex. As a consequence, lexical boost is often needed before a priming effect can be observed for this 
construction (As noted above, even for English native speakers, comprehension priming of RCs typically occurs when prime and target sentences use the same verb but may not occur at all when prime and target involve different verbs). For other structures where the possibilities are more balanced, lexically-independent priming in comprehension does occur in L1 comprehension (e.g., Kim et al., 2014; Pickering et al., 2013) and may be expected in L2. Future studies involving more structures are needed to test these possibilities.

In sum, this study showed that structural priming occurred in L2 online sentence comprehension, attesting to the universality of structural priming effects. Moreover, the pattern of results observed in this study is comparable to that observed in L1 comprehension priming, suggesting that similar lexically-specific mechanisms are involved in L1 and L2 structural priming for sentences with reduced relative clauses. Further research will be required to determine whether this pattern holds for other syntactic structures and for participants with a wider range of L2 proficiency. The present study represents an important step in understanding to what extent L2 processing is like L1 processing, and suggests that structural priming provides a useful tool for assessing syntactic representations and mechanisms underlying L2 sentence comprehension.

\section{Acknowledgements}

We thank Ding Min, Shen Huiyang, and Lei Siyu for help in running the experiment. We are grateful to Nick Ellis and two anonymous reviewers, whose comments and suggestions are extremely helpful in revising the manuscript.

\section{Declaration of conflicting interests}

The authors declare that there is no conflict of interest.

\section{Funding}

This research was supported by Humanities and Social Science Fund of the Chinese Ministry of Education to Hang Wei (15YJC740091) and Fang Yuan (16YJC740084), and the National Social Science Fund of China to Min Wang (13BYY069). 


\section{Notes}

1. Studies on L1 comprehension priming involving other syntactic structures reveal that priming can be reliably observed in the absence of lexical overlap between primes and targets (see Tooley and Traxler, 2010, for a review). It seems, therefore, that lexical overlap is not strictly necessary for priming of comprehension to occur.

2. Although a full list of experimental items was not available in Hui and Liu (2012), their sample sentences (like 2 and 3) showed that the thematic role of the disambiguating region and the animacy of the head noun varied across prime and target sentences. There is evidence that features such as thematic role and animacy affected structural priming during production (Bock and Loebell, 1990; Chang et al., 2003), and these features are typically held constant across prime and target sentences (as shown in 1a and 1b) in L1 comprehension priming (e.g., Tooley et al., 2009, 2014; Traxler and Tooley, 2008; Traxler et al., 2014).

3. The answers to two questions were accidentally exchanged, causing the actual score to be 48 .

4. Two participants in the Upperclassman group and four in the Freshman group scored below the exclusion cutoff of $80 \%$ accuracy in answering the comprehension questions and were excluded from further analyses, leaving 32 participants in each group. See Results for details.

5. In an ERP study that involved both MC and RC as primes (Ledoux et al., 2007), syntactic priming effects did not occur until the third word of the disambiguating by-phrase. In contrast, in ERP and eye-tracking studies that used only RC as primes (e.g., Tooley et al., 2009, 2014), syntactic priming occurred on the first word of the by-phrase.

6. Although we tested each participant's proficiency, only some of them wrote their Subject ID on the test paper, making it impossible to use the proficiency score as a continuous predictor.

7. We thank an anonymous reviewer for raising this issue.

\section{References}

Baayen RH, Davidson DJ, and Bates DM (2008) Mixed-effects modeling with crossed random effects for subject and items. Journal of Memory and Language 59: 390-412.

Bates DM, Kliegl R, Vasishth S, and Baayen RH (2015) Parsimonious mixed models. Available from arXiv:1506.04967.

Bates DM, Maechler M, Bolker B, et al. (2015) lme4: Linear mixed-effects models using Eigen and 
S4: R package version 1.1-9 [software package]. Retrieved from: https://cran.rproject.org/web/packages/lme4/index.html

Bernolet S, Hartsuiker RJ, and Pickering MJ (2013) From language-specific to shared syntactic representations: The influence of second language proficiency on syntactic sharing in bilinguals. Cognition 127: 287-306.

Bock K (1986) Syntactic persistence in language production. Cognitive Psychology 18: 355-87.

Bock K (1989) Closed-class immanence in sentence production. Cognition 31: 163-86.

Bock K and Loebell H (1990) Framing sentences. Cognition 35: 1-39.

Branigan HP and Pickering MJ (in press) An experimental approach to linguistic representation. Behavioral and Brain Sciences.

Chang F, Bock K, and Goldberg AE (2003) Can thematic roles leave traces of their places? Cognition 90: $29-49$.

Clahsen H and Felser C (2006a) Grammatical processing in language learners. Applied Psycholinguistics 27: 3-42.

Clahsen H and Felser C (2006b) How native-like is non-native language processing? Trends in Cognitive Sciences 10: 564-70.

Dell GS and Ferreira VS (2016) Thirty years of structural priming: An introduction to the special issue. Journal of Memory and Language 91: 1-4.

Fine AB and Jaeger FT (2016) The role of verb repetition in cumulative structural priming in comprehension. Journal of Experimental Psychology: Learning, Memory, and Cognition 42: 1362-76.

Foucart A, Martin CD, Moreno EM, and Costa A (2014) Can bilinguals see it coming? Word anticipation in L2 sentence reading. Journal of Experimental Psychology: Learning, Memory, and Cognition 40: 1461-69.

Hartsuiker RJ and Bernolet S (2015) The development of shared syntax in second language learning. Bilingualism: Language and Cognition 19: 1-16.

Hartsuiker RJ, Bernolet S, Schoonbaert S, Speybroeck S, and Vanderelst D (2008). Syntactic priming persists while the lexical boost decays: Evidence from written and spoken dialogue. Journal of Memory and Language 58: 214-38.

Hopp H (2010) Ultimate attainment in L2 inflection: Performance similarities between non native 
and native speakers. Lingua 120: 901-31.

Hopp H (2016) Learning (not) to predict: Grammatical gender processing in second language acquisition. Second Language Research 32: 277-307.

Hui K and Liu M (2012) Syntactic priming during English sentence comprehension in proficient Chinese learners of English. Journal of South China Normal University 5: 121-25.

Kaan E (2014) Predictive sentence processing in L2 and L1: What is different? Linguistic Approaches to Bilingualism 4: 257-82.

Kim CS, Carbary KM, and Tanenhaus MK (2014) Syntactic priming without lexical overlap in reading comprehension. Language and Speech 57: 181-95.

Kim Y and McDonough K (2008) Learners' production of passives during syntactic priming activities. Applied Linguistics 29: 149-54.

Ledoux K, Traxler MJ, and Swaab T (2007) Syntactic priming in comprehension: Evidence from Event-Related Potentials. Psychological Science 18: 135-43.

MacDonald MC, Pearlmutter NJ, and Seidenberg MS (1994) Lexical nature of syntactic ambiguity resolution. Psychological Review 89: 483-506.

Mahowald K, James A, Futrell R, and Gibson E (2016) A meta-analysis of syntactic priming in language production. Journal of Memory and Language 91: 5-27.

Nitschke S, Kidd E, and Serratrice L (2010) First language transfer and long-term structural priming in comprehension. Language and Cognitive Processes 25: 94-114.

Nitschke S, Serratrice L, and Kidd E (2014) The effect of linguistic nativeness on structural priming in comprehension. Language, Cognition and Neuroscience 29: 525-42.

Pickering MJ and Ferreira VS (2008) Structural priming: A critical review. Psychological Bulletin 134: 427-59.

Pickering MJ, McLean JF, and Branigan HP (2013) Persistent structural priming and frequency effects during comprehension. Journal of Experimental Psychology: Learning, Memory, and Cognition 39: 890-97.

Schoonbaert S, Hartsuiker RJ, and Pickering MJ (2007) The representation of lexical and syntactic information in bilinguals: Evidence from syntactic priming. Journal of Memory and Language 56: 153-71.

Tooley KM and Bock K (2014) On the parity of structural persistence in language production and 
comprehension. Cognition 132: 101-36.

Tooley KM, Swaab TY, Boudewyn MA, Zirnstein M, and Traxler MJ (2014) Evidence for priming across intervening sentences during on-line sentence comprehension. Language, Cognition and Neuroscience 29: 289-311.

Tooley KM and Traxler MJ (2010) Syntactic priming effects in comprehension: A critical review. Language and Linguistics Compass 4: 925-37.

Tooley KM, Traxler MJ, and Swaab T (2009) Electrophysiological and behavioral evidence of syntactic priming in sentence comprehension. Journal of Experimental Psychology: Learning, Memory, and Cognition 35: 19-45.

Traxler MJ and Tooley KM (2008) Priming in sentence comprehension: Strategic or syntactic? Language and Cognitive Processes 23: 609-45.

Traxler MJ, Tooley KM, and Pickering MJ (2014) Syntactic priming during sentence comprehension: Evidence for the lexical boost. Journal of Experimental Psychology: Learning, Memory, and Cognition 40: 905-18.

Weber K and Indefrey P (2009) Syntactic priming in German-English bilinguals during sentence comprehension. NeuroImage 46: 1164-72.

Yang H and Weir C (1998) Validation studies of the National College English Test. Shanghai: Shanghai Foreign Language Education Press.

\section{Appendix 1}

\section{Experimental stimuli}

S primes use the same initial verb (e.g., selected) as do the target sentences. D primes use an initial verb that is different from that in the target sentence (e.g., criticized vs. selected). The sentences were paired such that the $S$ prime was paired with the target on one experimental list and the D prime was paired with the target on a different list. Each S prime and target pair would then appear on a third list, where the prime sentence would appear in the target position and the target sentence would appear in the prime position. The same would occur for the $\mathrm{D}$ prime and target pairs on a fourth list.

1.

S Prime: The speaker selected by the group would be perfect for the program.

D Prime: The cook criticized by the customers used too much salt.

Target: The architect selected by the manager was educated at Yale.

2.

S Prime: The director watched by the cop was in poor health.

D Prime: The defendant examined by the psychologist was mentally stable.

Target: The mouse watched by the cat was hiding under a table.

3.

S Prime: The prisoner transported by the guards was planning an escape.

D Prime: The coach hugged by the player had an amazing career. 
Target: The hostages transported by the robbers were really terrified. 4.

S Prime: The troops attacked by the enemy suffered heavy losses.

D Prime: The parents worried by their son decided to talk to him.

Target: The army attacked by the rebels moved forward quickly.

5.

S Prime: The cowboys surrounded by the Indians prepared to fight.

D Prime: The worker punished by the boss cleaned up the workshop.

Target: The lions surrounded by the hunters paced nervously.

6.

S Prime: The driver stopped by the policeman had been drinking.

D Prime: The puppy washed by the old lady was covered in dust.

Target: The child stopped by the guard looked very upset.

7.

S Prime: The dog found by the hunter had a broken leg.

D Prime: The manager hired by the owner had to work 7 nights per week.

Target: The bird found by the scientist had beautiful features.

8.

S Prime: The child scolded by the mother went up the stairs.

D Prime: The pupil missed by the bus walked to school.

Target: The man scolded by the police was very embarrassed.

9.

S Prime: The driver injured by the truck had to go to the hospital.

D Prime: The student taught by her mother passed all examinations.

Target: The child injured by the dog breathed heavily.

10.

S Prime: The spy caught by the FBI agent disappeared forever.

D Prime: The lawyer preferred by the judge was always on time.

Target: The criminal caught by the police was put in prison.

11.

S Prime: The passengers delayed by the airplane were very angry.

D Prime: The landlord cheated by the couple was not paid any rent.

Target: The woman delayed by the taxi driver started to shout.

12.

S Prime: The students helped by the teacher were very thankful.

D Prime: The accountant fired by the firm was arrested for drug possession.

Target: The doctors helped by the nurses were extremely tired.

13.

S Prime: The deer killed by the train caused a large accident.

D Prime: The nurse cured by the medicine had been seriously ill.

Target: The zebra killed by the crocodile was crossing the river.

14.

S Prime: The boss hated by the workers was really mean.

D Prime: The girl kissed by the singer got so excited that she fainted.

Target: The doctor hated by his patients had a bad temper.

15.

S Prime: The suspect accused by the judge had taken bribes.

D Prime: The pupil praised by the teacher studied extra longer that week.

Target: The politician accused by the media was fairly defensive.

16.

S Prime: The man lifted by the elephant was very nervous.

D Prime: The girl pulled by her father wanted to stay at the zoo.

Target: The monkey lifted by the trainer was hoping for a banana.

17.

S Prime: The player praised by the team leader was soon offered a scholarship.

D Prime: The politician accused by the media was fairly defensive.

Target: The pupil praised by the teacher studied extra longer that week.

18.

S Prime: The mother kissed by the boy gave him a hug in return.

D Prime: The doctor hated by his patients had a bad temper. 
Target: The girl kissed by the singer got so excited that she fainted. 19.

S Prime: The principal fired by the school had been missing work.

D Prime: The doctors helped by the nurses were very tired.

Target: The accountant fired by the firm was arrested for drug possession.

20.

S Prime: The customer cheated by the company refused to purchase their product.

D Prime: The woman delayed by the taxi driver started to shout.

Target: The landlord cheated by the couple was not paid any rent.

21.

S Prime: The waitress preferred by the princess got a large tip.

D Prime: The criminal caught by the police was put in prison.

Target: The lawyer preferred by the judge was always on time.

22.

S Prime: The girl taught by the chef made a wonderful cake.

D Prime: The child injured by the dog breathed heavily.

Target: The student taught by her mother passed all examinations.

23.

S Prime: The singer missed by the conductor had a sore throat.

D Prime: The man scolded by the police was very embarrassed.

Target: The pupil missed by the bus walked to school.

24.

S Prime: The baker hired by the market made eight different kinds of bread.

D Prime: The bird found by the scientist had beautiful features.

Target: The manager hired by the owner worked seven nights per week.

25.

S Prime: The girl washed by her mother was very cold.

D Prime: The child stopped by the guard looked very upset.

Target: The puppy washed by the old lady was covered in dust.

26.

S Prime: The staff punished by the manager had to work three extra hours.

D Prime: The lions surrounded by the hunters paced nervously.

Target: The worker punished by the boss cleaned up the workshop.

27.

S Prime: The captain worried by the storm stayed awake all night long.

D Prime: The army attacked by the rebels moved forward quickly.

Target: The parents worried by their son decided to talk to him.

28.

S Prime: The girl hugged by the grandparent felt safe and warm.

D Prime: The spy transported by the police was very worried.

Target: The coach hugged by the player had an amazing career.

29.

S Prime: The player examined by the doctor was allowed to finish the game.

D Prime: The mouse watched by the cat was hiding under a table.

Target: The defendant examined by the psychologist was mentally stable.

30.

S Prime: The dancer criticized by the audience had poor performance.

D Prime: The architect selected by the manager was educated at Yale.

Target: The cook criticized by the customers used too much salt.

31.

S Prime: The lawyer pulled by the boy made a foolish decision.

D Prime: The monkey lifted by the trainer was hoping for a banana.

Target: The girl pulled by her father wanted to stay at the zoo.

32.

S Prime: The doctor cured by the specialist went back to work.

D Prime: The zebra killed by the crocodile was crossing the river.

Target: The nurse cured by the medicine had been seriously ill. 


\section{Appendix 2}

\section{Details of the mixed-effects analyses and model-fitting procedure}

We entered the following variables as fixed effects: priming, verb repetition, and education level. In addition, we also included the length of each region, comprehension question accuracy, and stimulus order, and the interaction between priming and stimulus order (to capture potential cumulative priming effects). Hence, the fixed effects used for the LMEMs are:

$$
\begin{aligned}
\log (\mathrm{RT}) \sim \text { Priming } * \text { Verb repetition } * \text { Education level }+ \text { Region length }+ \text { Stimulus order } \\
+ \text { Accuracy }+ \text { Priming }: \text { Stimulus order }
\end{aligned}
$$

For the random effects structure of the subjects, we included random intercepts, random slopes, and full covariances by Priming and Verb repetition, and their interaction; for the random effects of items, we included random intercepts, random slopes, and full covariances by Priming, Education level, and Verb repetition, and their interaction. The random effects structure was determined by adapting the procedure described in Bates, Kliegl, et al. (2015). The procedure was applied in the following way:

1. We began with a maximal random effects structure;

2. We removed terms under the following conditions:

- Model failed to converge

- Covariances at edge values: [-1 1 1]

- Variances at edge values: 0

3. Where there were multiple possible back-off paths, we removed terms in the following order:

- Covariances first (e.g., "Zero Correlation-Parameter” model)

- Then random intercepts

- Then random slope interactions

- Then random slope main effects

After arriving at the model that both converged and lacked edge values, terms were added back in individually. If the resulting model also converged, the terms were retained. Categorical predictors were converted to vectors for use with “double- bar" || syntax for removing random correlation parameters. The procedure was conducted on the Verb region and checked against the $P P$ and post-PP regions. The maximum number of iterations used to fit each model was set to 30,000 . Details of the model-fitting procedure are as follows. 
1. Starting on the Verb region, the maximal model failed to converge. We then fitted a Zero-

Correlation Parameter (ZCF) LMEM for the random terms.

2. The resulting model showed problematic edge-case variances $=0$ for:

- Priming (by-subjects)

- Education level (by-items)

- Education level : Priming interaction (by-items)

- Education level : Verb repetition interaction (by-items)

- Education level : Priming : Verb repetition interaction (by-items)

We removed these four random slope terms.

3. This model converged without any random covariances $=[-11]$ or variances $=0$. We then added back in random correlations parameters.

4. This expanded model failed to converge and showed a problematic Verb repetition : Priming (bysubjects) covariance $=1$. We removed this by-subjects covariance component.

5. This model converged but shows a problematic Verb repetition (by-subjects) variance $=0$. We removed this random slope.

6. This model converged. We added back in the by-participants random slope for the Priming : Verb repetition interaction.

7. This model converged without any problematic indications in the random variance-covariance matrix. This is the final random effects structure for Verb region:

$$
\begin{aligned}
& (1+\text { Verb repetition } * \text { Priming } \mid \text { Subject })+ \\
& (1+\text { Priming }+ \text { Verb repetition }+ \text { Priming } * \text { Verb repetition } \mid \text { Item })
\end{aligned}
$$

8. Applying the model from (7) to the PP region converged, but shows a by-subjects interaction covariance $=1$. We dropped the by-subjects covariances and re-tested against the PP region.

9. The result converged, but now shows a problematic by-items covariance $=1$. We dropped the byitems covariance parameters.

10. The resulting model converged, but showed zero variance for the by-subjects Priming : Verb repetition random slope. We dropped this component.

11. The resulting model converged and did not show any problematic variances values. We added back in by-items covariance components.

12. The resulting model converged and did not show any problematic values in the random variance- 
covariance matrix. This is the final random effects structure for the PP region:

$(1 \mid$ Subject $)+(1+$ Priming + Verb repetition + Priming $*$ Verb repetition $\mid$ Item $)$

13. We finally moved to the post-PP region using the model from (7) above. This model converged and showed no problematic values in the variance-covariance matrix. We retained this model for the final random effects structure in the post-PP region:

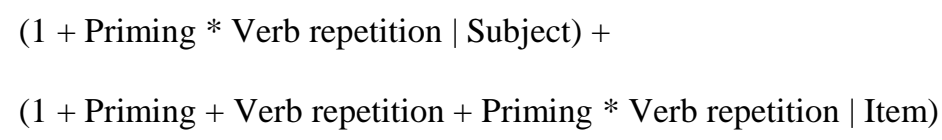

Note that the fixed-effect estimates and uncertainties were qualitatively the same across all of the models that were fit in this procedure. 